8

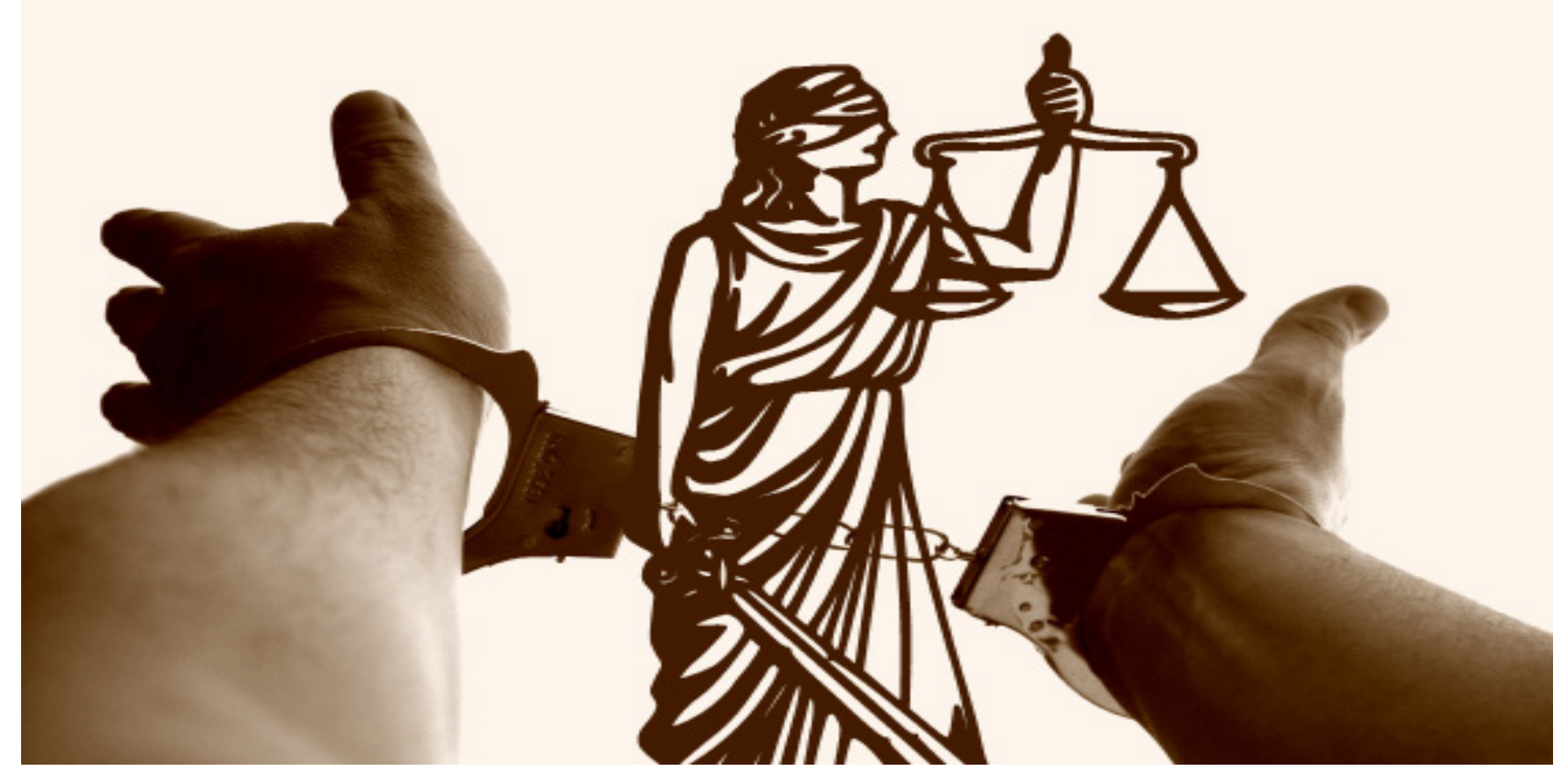

\title{
Antropología criminal en Colombia: Análisis de sus principales obstáculos
}

\section{Criminal anthropology in Colombia: Analysis of its main obstacles}

Elizabeth Ramírez-Llerena

Doctorado en Sociologia Jurídica e Instituciones Políticas, ramirez@unitecnar.edu.co, https://orcid.org/0000-0002-4462-8989, Fundación Universitaria Antonio de ArévaloUnitecnar, Cartagena de Indias, Colombia. 
Resúmen

El presente trabajo abordará cuáles son los principales obstáculos de la antropología criminal en Colombia. Haciendo un examen crítico y exhaustivo de la producción antropológica criminal del país, cuestionando la ausencia de aportes en esta materia, la carencia de creatividad y la poca evolución de la antropología criminal. Desde el punto de vista de la sociología del conocimiento, emprende un análisis sobre la manera como se produce el saber criminológico o, mejor, sobre la forma cómo se reproduce, al igual que estudia el rol de los antropólogos criminales del país. También evalúa los problemas que significa la ausencia de una comunidad académica y de sentido crítico frente a la poca producción.

Palabras clave: Antropología criminal, ciencia, criminología, Escuela positivista, aportes, Estado, política criminal, delincuente.

\section{Abstract}

This paper will address what are the main obstacles of criminal anthropology in Colombia. It makes a critical and exhaustive examination of the country's criminal anthropological production, questioning the absence of contributions in this area, the lack of creativity and the little evolution of criminal anthropology. From the point of view of the sociology of knowledge, it undertakes an analysis of the way in which criminological knowledge is produced or, better still, how it is reproduced, as well as studying the role of the country's criminal anthropologists. It also assesses the problems of the absence of an academic and critical community in the face of low production.

Keywords: Criminal anthropology, science, criminology, positivist school, contributions, state, criminal policy, criminal. 


\section{0}

Introducción

De acuerdo a lo planteado por [1] "la Antropología criminal es la ciencia que estudia los caracteres físico-psíquicos del hombre delincuente, y, sobre la base de éstos, juntamente con las influencias del ambiente $y$ de las circunstancias". En pocas palabras es el estudio de los seres humanos desde una perspectiva biológica, social y humanista, estudia la personalidad del delincuente; Cesare Lombroso es su máximo exponente; él afirmaba que el hombre estaba determinado a ser delincuente por sus características físicas, fueran estas heredadas o no.

De ahí que se contemplara la existencia de causas pre constituido para que una persona perpetrara un crimen, motivo por el cual fue aceptada la denominada peligrosidad de un individuo, entonces la antropología criminal estudia al hombre de manera biológica y sociocultural del delincuente [2]. Por otra parte, uno de los principales y grandes problemas con el que se encuentran las personas especializadas en antropología criminal, es el que hace alusión a la carencia y escasez, por no decir, la inexistencia absoluta de investigaciones que marque el camino a seguir en su trabajo.

Desde hace mucho tiempo se viene criticando la carencia de aportes que hay en materia de antropología criminal en Colombia; Luis Jiménez de Asúa afirma que "en casi todas partes los positivistas se hacen - valga la frase- escolásticos y citan a Ferri y Garófalo como podían invocar los nombres de Carrara o de Berner. No investigan la realidad delincuente, ni estudian las características del criminal ni las causas del delito [3]. Sólo citan lo que hicieron, hace sesenta años, aquellos revolucionarios de su tiempo." Hoy en día la situación sigue casi igual, no hay investigaciones, la producción académica es escasa.
Dicho lo anterior, el presente trabajo investigará cuáles son las razones y los obstáculos a los cuales se enfrentan los especialistas en antropología criminal en Colombia.

\section{Materiales y métodos}

Se utilizó el método cualitativo, ya que, dicha problemática ha sido abarcada con una estrategia metodológica histórica, dialéctica y analítica, basada en una amplia observación, recopilación y revisión de fuentes documentales de autores reconocidos en Colombia y en exterior; entrevistas a profesionales en esta materia etc. Lo anterior, con el fin de abordar el problema de esta investigación representado en la necesidad de descifrar los principales obstáculos que influyen en los procesos de desarrollo del saber antropológico que inciden en la construcción social imaginaria acerca de la criminalidad y los criminales en Colombia.

Esta investigación es descriptiva porque se pretende llegar a conocer las situaciones, costumbres y actitudes predominantes a través de la descripción exacta de las actividades, objetos, procesos y personas. Su meta no se limita a la recolección de datos, sino a la predicción e identificación de las relaciones que existen entre dos o más variables. Los investigadores no son meros tabuladores, sino que recogen los datos sobre la base de una hipótesis o teoría, exponen y resumen la información de manera cuidadosa y luego analizan minuciosamente los resultados, a fin de extraer generalizaciones significativas que contribuyan al conocimiento.

Las diversas fuentes de información utilizadas para formular los resultados del presente artículo, se estructuró a partir de los siguientes recursos metodológicos:

1. Análisis de publicaciones y estudios existentes la antropología criminal en Colombia. 
2. Entrevista con miembros de la fiscalía y antropólogos nacionales.

3. Fuentes de información citadas en los resultados de la investigación de diversos autores.

\section{Resultados}

Como se mencionó anteriormente, la antropología criminal es una rama de la criminología que se encarga de estudiar las características físicas y mentales de los criminales (personalidad del criminal), así como los factores sociales y medioambientales que influyen en la conducta criminal, siendo Cesare Lombroso el máximo exponente de esta ciencia razón por la cual, desde los tiempos de este autor e incluso en la actualidad, la antropología criminal colombiana ha seguido la corriente de la escuela positivista. Ahora bien, esta presenta algunos obstáculos que han venido transcurriendo por más de medio siglo según Luis Jiménez de Asúa.

Principales obstáculos de la antropología en Colombia

\section{Carencia de material académico e} investigaciones nuevas

La realidad de la delincuencia además de las características del criminal y de las causas del delito cambió a fines de los años 80, la antropología criminal en Colombia viró hacia el estudio de la problemática de operación del control penal transformando esta vertiente en un problema mayoritaria, descrito como el análisis de los fenómenos de la divergencia social y del control social penal [3]. Pero con todo esto, las acciones sociales calificadas como, criminales en otras palabras criminalidad o el control penal son el grueso de los antropólogos colombianos de ahora y de antes, pero que sus actitudes se limitan a citar autores, a repetir las teorías de otros autores, permanecen sin investigar, sin discutir, sin intentar hacer aportes teóricos, es decir, solo quedan satisfechos a repetir lo que dicen autores extranjeros convertidos en lo que Jiménez Asúa llamó como "escolásticos".

Esto se ha convertido en uno de los principales obstáculos para el buen desarrollo de la antropología criminal, debido a que hay carencia de nuevos aportes en virtud de desarrollos metodológicos. Ejemplo de esto, son autores [4] con su artículo "Aproximaciones a la antropología criminal desde la perspectiva de Lombroso" artículo que hace énfasis en la teoría de Lombroso, Ricardo Hernán Medina Rico "El Delincuente Predeterminado: De la criminología positiva italiana a la neurocientificidad", Jaime Samudio Diaz "El comportamiento criminal en Colombia" Ervyn Hermilzon Norza, Luz S. Rodríguez, Sergio Useche y José Ignacio Ruiz Pérez "Investigación Criminológica: Teorías y Modelos Explicativos de la Criminología" esta investigación se basa en las teorías de Lombroso [5]". Los fundamentos de la sociología criminal". Los anteriores artículos son un gran ejemplo de la poca creación de material nuevo en el país, estos se basaron en copiar y repetir los aportes realizados por la escuela positivista italiana, es decir, se copian y repiten cosas desuetas o bastante desactualizadas.

Con todo esto, la mayor producción entorno a la criminalidad en Colombia y los problemas estudiados por la antropología han venido de otras disciplinas. [3] "La economía, y la sociología, sobre todo, pero también la historia, la criminología y la psicología, han generado una producción bastante extensa en términos cuantitativos, pero así mismo destacada en un plano cualitativo. Ella se ha ocupado del conflicto armado, del homicidio, del tráfico de drogas, del aborto, del contrabando, de la justicia penal, de las políticas penales, de la corrupción, etc.". Pero a ciencia cierto, desde 


\section{2}

el punto de vista del conocimiento, no importa mucho si la divergencia social de relevancia penal y al control social penal provienen de otras disciplinas, lo que interesa son sus contribuciones, aquellas que rompen con esas tendencias mediocres a la "escolástica" que llamaba Jiménez Asúa, en los cuales se encuentran la mayoría de antropólogos criminales en Colombia. Con el fin de combatir a los escolásticos, lo cuales tanto daño le han hecho a esta disciplina, por esto convendría que en los escenarios universitarios se enseñara a los estudiantes, aquellos que serán finalmente los profesionales de la antropología criminal en Colombia a identificar trabajos, textos de gran calidad, revisar sus bibliografías, examinar si la misma en un grado significativo está actualizada; que ayuden a mejorar aquellos obstáculos que impiden el buen funcionamiento de la antropología criminal en Colombia.

2. Falta de datos estadísticos y mal recopilamiento de la información

Otro de los obstáculos a los que se enfrenta los Antropólogos criminales en Colombia, es a la falta de información cualitativa-cuantitativa, que les permita llevar a cabo investigaciones. La disponibilidad de información estadística y su calidad destacan por su baja calidad, la falta de armonización y de criterios de unificación de las diferentes fuentes de información, la ausencia de datos de victimización, entre otros. Como consecuencia de lo anterior, la falta de encuestas y estadísticas de victimización impiden examinar la percepción de la ciudadanía, se pierde información valiosa con los delitos que son cometidos, pero, no son denunciados.

Esta problemática conduce al desconocimiento de uno de los principales objetos de la antropología criminal: la criminalidad. Esto supone, que no se conozcan con exactitud la cifra de los delitos cometido y su modus operandi. No hay en Colombia la capacidad tecnológica y personal especializado para recopilar la información para su respectivo análisis, las instituciones encargadas de recopilar la información están fallando. Es evidente la carencia de investigaciones a profundidad, que abarquen las características delictivas en relación con las condiciones socioeconómicas, institucionales y culturales, [6]"las costumbres, las tradiciones, el folklore y las creencias juegan un papel importante en las medidas de prevención, ya que suponen a su vez controles sociales" En efecto, la falta de información y datos producen inconvenientes a la de desarrollar las medidas de control social.

Por otra parte, cuando existe información cuantitativa, se presentan lagunas en las estadísticas, producidas por el mal manejo de la información, hay un margen de error que se originan de las mismas fuentes de información, por muchos factores como inadecuada capacitación del personal encargado de tomar dicha información, deficiencias técnicas de los formularios utilizados para plasmar la información. [7] "los guarismos pueden conducir a conclusiones vulnerables o equivocas, la pretendida homogeneidad (unidad estadística) pero ayuda mucho a señalar los puntos álgidos, crecimiento, decrecimiento, conexidad, amplitud, extensión, saturación e intensidad del delito, con este reconocimiento, es posible proceder con éxito tanto en la prevención, como en la represión y aplicar lentes científicas desentrañando de la masa estadística aquellos gérmenes patógenos perturbadores del cuerpo social" En pocas palabras nos dice, que la estadística criminal es arma de doble filo dado que, puede conducir a resultados erróneos.

Las principales consecuencias de la falta de datos estadístico es; La construcción de una política criminal exitosa, [8]" Esta falta de investigación de los delitos y las respuestas 
sociales a la delincuencia en el mundo en desarrollo significa que la política criminal ha sido dictada por el material que se encuentra en el dominio de los organismos de justicia penal [aun cuando] muchos de los datos tienen que ser abordados con precaución debido a la posibilidad de lagunas, incoherencias y errores" Para desarrollar una política criminal exitosa que dirija todos los esfuerzos institucionales a evitar, en la medida de lo posible, la ocurrencia de los delitos, y de esta manera preservar los derechos ciudadanos. Para esto, se necesitan una base sólida de información cuantitativa, que ayuden a fortalecer esta. Otra consecuencia es desarrollar perfiles criminológicos; desde el enfoque cuantitativo y estadístico, el estudio de grandes muestras de delitos cometidos y delincuentes permite identificar variables que facilitan el desarrollo de perfiles. El hecho de que se tomen en cuenta datos de casos ya resueltos y que se realicen análisis estadísticos para descubrir patrones que se repiten en diversos casos, permite predecir con mayor precisión las características de delincuentes desconocidos.

3. El enfoque de la antropología criminal en Colombia, no va acorde a la realidad del país

De acuerdo a lo planteado por [9] "hoy la acepción Antropología criminal ha perdido su significado tradicional. Prácticamente ya no se utiliza en Criminología; a no ser para referirse a la investigación en el positivismo criminológico, especialmente a Lombroso" La antropología criminal actualmente en Colombia, ha quedado olvidada, a tal punto que ya no se usa casi. La perspectiva que se le ha dado a la antropología criminal en el país, es el estudio de los rasgos fisiológicos que hacen que una persona delinca; en la actualidad estaría fuera de lugar, hablar de rasgos fisiológicos, que identifiquen al delincuente. La mayoría de investigaciones que se encuentran de antropología criminal en Colombia, van enfocada a los postulados de Lombroso, Ejemplo: Omar Huerta Diaz con su artículo "Aproximaciones a la antropología criminal desde la perspectiva de Lombroso" [10]". La antropología no ha estado al frente del estudio de la violencia colectiva, el terrorismo y especialmente, la violencia en sociedades estatales. Esto ha sido el resultado en parte de que sus métodos y teorías dependen de meses o años en un campo definido hasta hace recientemente como una comunidad pequeña y auto contenida, que no incluía el estado." como se puede observar, el enfoque de la antropología criminal en Colombia, no va acorde con la realidad del país.

El enfoque de la antropología criminal en Colombia, debe ir acorde a la realidad del país, y encaminarse, como la disciplina que tiene al delincuente $\mathrm{y}$ al acto delictivo como centro de estudio. De este modo, debe centrarse en aspectos como la delincuencia, instituciones carcelarias, medidas de castigo y reconstrucción, conflicto armado, centros de menores, teorías de la desviación, edades del delito, etnografías sobre las cárceles, casas de convivencia, delincuencia juvenil, pandillas, mafias, etc. Para lograr este objetivo, debe apoyarse de Antropología Social, para formar una base sólida de esta disciplina.

Dicho lo anterior, podemos comenzar citandoa antropólogos criminales internacionales, como Wacquant, que mediante sus investigaciones acerca de la pobreza urbana y las clases marginadas; autores como [11] con sus análisis sobre el consumo y sus estructuras o el sociólogo Becker con sus estudios sobre la desviación, son autores en que los antropólogos colombianos, deben basarse para darle un enfoque a esta disciplina, acorde a la realidad. Por otra parte, en el ámbito local, las investigaciones recientes en Colombia se van alejando de las teorías de Lombroso, y dándole una perspectiva a la antropología criminal, más acorde a la realidad. Alejandro Castillejo-Cuéllar, con su investigación. "La localización del daño: etnografía, espacio, 


\section{4}

y confesión en el escenario transicional colombiano". El Ministerio de Justicia y del Derecho" Fortalezas y debilidades de la investigación y judicialización criminal del delito de trata de personas", Juan Pablo Vera Lugo "Antropología y estudios de la violencia en Colombia: en busca de una perspectiva crítica". Estas investigaciones, apoyadas de la antropología social, han permitido comenzar a formar una base teórica sólida, que permite construir un tronco solido a esta disciplina.

En el dominio de los organismos de justicia penal [aún cuando] muchos de los datos tienen que ser abordados con precaución debido a la posibilidad de lagunas, incoherencias y errores" Para desarrollar una política criminal exitosa que dirija todos los esfuerzos institucionales a evitar, en la medida de lo posible, la ocurrencia de los delitos, y de esta manera preservar los derechos ciudadanos. Para esto, se necesitan una base sólida de información cuantitativa, que ayuden a fortalecer esta. Otra consecuencia es desarrollar perfiles criminológicos; desde el enfoque cuantitativo y estadístico, el estudio de grandes muestras de delitos cometidos y delincuentes permite identificar variables que facilitan el desarrollo de perfiles. El hecho de que se tomen en cuenta datos de casos ya resueltos y que se realicen análisis estadísticos para descubrir patrones que se repiten en diversos casos, permite predecir con mayor precisión las características de delincuentes desconocidos.

4. Falta de información acerca de antropología criminal

Para comenzar, la antropología criminal en Colombia, está llena de huecos y vacíos, los cuales afectan su práctica en el país. Uno de los principales obstáculos de esta disciplina, es la falta de información e investigaciones, en la cual basarse para realizar nuevos trabajos. Su falta de utilización en entidades Universitarias, donde solo se tiene la antropología criminal como un curso dentro de una carrera ejemplo: Tecnar.
Hace falta autores, especialistas en Colombia y una mayor inversión de parte del gobierno; [12], los principales obstáculos a los que él se enfrentan, a la hora de realizar investigaciones en materia antropológica criminal son: El desplazamiento hacia los lugares, ya que la seguridad es lo más importante y este se convierte en un problema muy grave, por ejemplo en los casos de los Paramilitares se debe ir con un grupo de trabajo de criminalística de campo y a veces falla la policía y vehículos. Muchas veces no cuentan con las condiciones óptimas para llegar, porque los sitios son muy inhóspitos porque se encuentran en sitios muy alejados del perímetro urbano, y, por ende, para llegar allá se necesita de mucha seguridad; también en la parte de la logística, haciendo referencia a la escasez de vehículos.

Asimismo, el Instituto Colombiano de Antropología e historia ha tenido un rol bastante pasivo frente a las necesidades de investigación relacionadas con la antropología criminal, aportando nada en la coyuntura colombiana. Por otra parte, la criminología al no ser una ciencia autónoma e independiente en Colombia, hace que la antropología criminal en Colombia pase desapercibido y pierde peso. En Colombia la criminología no es una ciencia independiente como en otros países, no existen en el país universidades, que cuenten con la criminología como una carrera profesional, a diferencias de países como México, donde hay más de 23 centros académicos que tienen la Criminología como carrera profesional.

Por otra parte, [13] Antropólogo social de la universidad del cauca, nos dio a entender que la antropología criminal en el país no se está dando debido a la falta de presupuesto, laboratorios que el país ofrece al especialista de esta disciplina, sea aplicada, por supuesto al no tener dinero no habrá laboratorios para su aplicación, en Colombia las faltas de aportes científicos para esta son vacíos que aún no llena el país. 


\section{Conclusiones}

Para finalizar, son diversos los obstáculos a los que se enfrentan los antropólogos criminales en Colombia, entre los cuales se encuentran:

1.Carencia de material académico e investigaciones nuevas.

2.Falta de datos estadísticos y mal recopilamiento de la información.

3.El enfoque de la antropología criminal en Colombia, no va acorde a la realidad del país.

4. Falta de información acerca de antropología criminal.

Son diversos los motivos por los cuales se producen estos obstáculos, la falta de profesionales, laboratorios, presupuesto, de universidades y el apoyo del gobierno para realizar investigaciones, son algunos de esto.

Existe un desconocimiento generalizado sobre la antropología criminal, lo que genera como efecto negativo de que sea una disciplina poca llamativa para los estudiantes. De acuerdo con la revisión realizada, es evidente la necesidad de abordaje esta problemática, se hace imposible desconocer su importancia y urgente abordaje, darle la importancia que la antropología criminal se merece, para que brinde a los criminólogos colombianos las herramientas necesarias para prevención de conductas punibles. Por ende, la mayor consecuencia de esta problemática es el desarrollo de una política criminal exitosa, ya que, es insuficiente para ofrecer respuestas efectivas, que ayuden a prevenir los delitos [14].

Por ende, avanzar por el camino de la construcción de una autentica comunidad académica, que disponga de sentido crítico y no tema emplearlo parecería una estrategia indispensable para revertir el problema que presenta la antropología en Colombia. Estimular la creatividad, el espíritu crítico y la imaginación, a la par que se combate la escolástica y el dogmatismo, en especial en las nuevas generaciones, sería igualmente requerido. Además, el rol de los académicos en la investigación y la producción científica debe cambiar radicalmente, ya que esto afecta a las instituciones de educación superior nacional al mismo tiempo que crea mal formación de aquellos estudiantes, que finalmente se convierten en profesionales, que con su práctica afectan y generan obstáculos a la antropología criminal en Colombia [15-16].

\section{Referencias}

[1] G. Bustillo-Blancas, "Antropología Criminal", Plataforma Digital de Derecho, Ciencias Sociales y Humanidades, 2018. [En línea]. Disponible en: https://leyderecho.org/ antropologia-criminal/

[2] O. Huertas-Díaz, "Aproximaciones a la antropología criminal desde la perspectiva de Lombroso", Revista criminalidad, vol. 53, no. 1, pp. 293-306, 2011

[3] G. García, "El estado de la criminología en Colombia a propósito de la crítica de Luis Jiménez de Asúa", Dialogo de saberes, no. 37, pp. 105-114, 2012.

[5] M. García, Los fundamentos de la sociología criminal. Bogotá D.C. Universidad Nacional de Colombia, 2017

[6] J. Vera, "Antropología y estudios de la violencia en Colombia: en busca de una perspectiva crítica", Revista colombiana de antropología, vol. 51, n.0 1. pp. 245-269, 2015.

[7] EQUITAS, Fortalecer el gremio científico forense en Colombia Balance y Retos, 2011. [En línea]. Disponible en: https:// 


\section{6}

www.equitas.org.co/sites/default/files/ biblioteca/2011FortalecerGremioFINAL_inv. pdf

[8] I. Alcalde, "La antropología criminal dentro de la antropología social. Un nuevo enfoque para su revitalización", Revista Nuevas Tendencias en Antropología, $n^{\circ}$ 8, pp. 1-20, 2017

[9] A. Gómez, G. García, El futuro de la criminología crítica, Colección ius penal 9. Bogotá, D.C. Editorial Universidad Católica de Colombia, 2015

[10] R. Naranjo y M. Olarte, Criminología y política criminal con enfoque psicojuridico dirigido a niños en edad pre - escolar. Bogotá D.C, 2017

[11] Z. Bauman, Trabajo, consumismo y nuevos pobres, 2017. [En línea]. Disponible en: https:// gedisa.com/gacetillas/302484.pdf

[12] R. Medina, "El Delincuente Predeterminado: De la criminología positiva italiana a la neurocientificidad", Criterio Jurídico, vol 15, no. 2 pp. 77-94, 2016

[13] F. Benavides, Criminología en Colomia. Colección Criminología. Editorial Ibáñez. Bogotá, D.C, 2017

[14] A. Romero, N. Lizarazo y L. Camacho, Crimen y política pública criminal: elementos para la configuración del observatorio de política criminal. Bogotá: Ministerio de Justicia y del Derecho, 2017

[15] E. Norza, L. Merchan, L. Morales y D. Meléndez, Perfilación criminológica: una revisión de la literatura y su aplicación en la investigación criminal en Colombia. Bogotá, D. C, 2015
[16] S.Y Garcia Salazar, J.M. Rojas-Ovalle, y E.J Barrientos Monsalve, "Alcance de las responsabilidades de las fiscalías de Venezuela y Colombia en la franja fronteriza "estudio de caso por homicidios", Mundo Fesc, vol. 8, n. ${ }^{\circ}$ 16, pp. 78-85, ene. 2019 